\title{
Organic photostimulated luminescence
}

Manabu Sakurai, ${ }^{1}$ Ryota Kabe, ${ }^{2,3, *}$ Masaaki Fuki, ${ }^{4}$ Zesen Lin, ${ }^{2,3}$ Kazuya Jinnai, ${ }^{3,5}$ Yasuhiro Kobori, ${ }^{1,4}$ Chihaya Adachi, ${ }^{3,5,6}$ and Takashi Tachikawa ${ }^{1,4, *}$

${ }^{1}$ Department of Chemistry, Graduate School of Science, Kobe University, 1-1 Rokkodai-cho, Nada-ku, Kobe 657-8501, Japan.

${ }^{2}$ Organic Optoelectronics Unit, Okinawa Institute of Science and Technology Graduate University, 1919-1 Tancha, Onna-son, Kunigami-gun, Okinawa 904-0495, Japan.

3 JST, ERATO ADACHI Molecular Exciton Engineering Project, Kyushu University, 744 Motooka, Nishi-ku, Fukuoka 819-0395, Japan.

${ }^{4}$ Molecular Photoscience Research Center, Kobe University, 1-1 Rokkodai-cho, Nada-ku, Kobe 657-8501, Japan.

${ }^{5}$ Center for Organic Photonics and Electronics Research (OPERA), Kyushu University, 744 Motooka, Nishi-ku, Fukuoka 819-0395, Japan.

${ }^{6}$ International Institute for Carbon Neutral Energy Research (WPI-I2CNER), Kyushu University, 744 Motooka, Nishi-ku, Fukuoka 819-0395, Japan.

* ryota.kabe@oist.jp (R.K.); tachikawa@port.kobe-u.ac.jp (T.T.) 
Photostimulated luminescence, which allows energy or data to be stored and released using electromagnetic waves as both the input and output, has attracted considerable interest in the fields of biomedical and informatics technologies, but this phenomenon is mostly limited to solid inorganic materials. ${ }^{1-4}$ Here, we report photostimulated luminescence from purely organic blend films composed of electron donor, acceptor, and trap/emitter molecules. In the films, charges are accumulated as radical ions by ultraviolet light irradiation and then extracted by near infrared light irradiation to produce visible light. Films are capable of multiple cycles $(>10$ times) of organic photostimulated luminescence, which was still observable from films left in the dark at room temperature for one week after excitation, and emission color could be varied by changing the trap/emitter molecules. These findings will broadly impact existing applications and provide new prospects for innovative flexible devices.

Organic semiconductors ${ }^{5-8}$ and soft crystals $^{9}$ (e.g., photochromic compounds, ${ }^{10}$ porous coordination polymers/metal-organic frameworks, ${ }^{11}$ and metal halide perovskites ${ }^{12,13}$ ) are promising materials for future technologies such as wearable optoelectronic devices and ultrahigh density data storage owing to their structural diversity, mechanical elasticity, low cost, and facile processing. In addition, optical and electronic properties of these materials can be tailored through molecular design to yield efficient light emission or electric power conversion with tunable band gap energies.

Recently, organic long persistent luminescence (LPL) systems producing a glow-in-thedark effect have been demonstrated by retaining long-lived charge-separated states over periods of up to an hour at room temperature in blend films consisting of two or more organic compounds. ${ }^{14,15}$ These systems are free of rare metals and can be fabricated via simple meltcasting at relatively low temperatures or solution processing at room temperature. Since then, 
a variety of organic LPL materials have been developed by combining different electron donor/acceptor pairs. ${ }^{16-19}$

In this paper, we demonstrate purely organic photostimulated luminescence (PSL) over a wide range of colors utilizing ultraviolet (UV) and near-infrared (NIR) light for multiple write-in and read-out cycles, respectively, by adding a molecule with dual roles as an electron trap and light emitter to an organic LPL system. As a model system, we first explore a ternary blend film of electron donor (4,4',4"-tris[(3-methylphenyl)phenylamino]triphenylamine; mMTDATA) (1 mol\%), electron acceptor (2,8-bis(diphenylphosphoryl)dibenzo[b,d]thiophene; PPT), and electron trap/emitter (5,6,11,12-tetraphenyltetracene; Rb) (1 mol\%, unless otherwise noted) (Fig. 1a).

Excitation of the film with UV light induces formation of charge-separated states between m-MTDATA and PPT, resulting in a green-emitting charge transfer (CT) excited state or exciplex (Fig. 1b). ${ }^{20}$ The excitation energy of the $\mathrm{CT}$ excited state is transferred to $\mathrm{Rb}$ via Förster resonance energy transfer (FRET), resulting in persistent orange emission. These processes are analogous to the organic LPL phenomena reported previously. ${ }^{15}$

At the same time, a portion of the electrons are captured by neutral $\mathrm{Rb}$ molecules, which have lowest unoccupied molecular orbitals (LUMOs) that are approximately $0.8 \mathrm{eV}$ lower than that of PPT, to form the long-lived radical anions of $\mathrm{Rb}\left(\mathrm{Rb}^{\bullet-}\right)$. Since $\mathrm{Rb}^{\bullet-}$ possesses strong absorption bands in the NIR region, NIR excitation can induce electron transfer from doublet excited $\mathrm{Rb}^{\bullet-}$ to neighboring PPT molecules, thereby producing mobile electrons in the PPT film for formation of CT states and subsequent FRET to Rb. In this way, the trap/emitter can enable PSL and the optical writing and reading of data in organic films. 
a
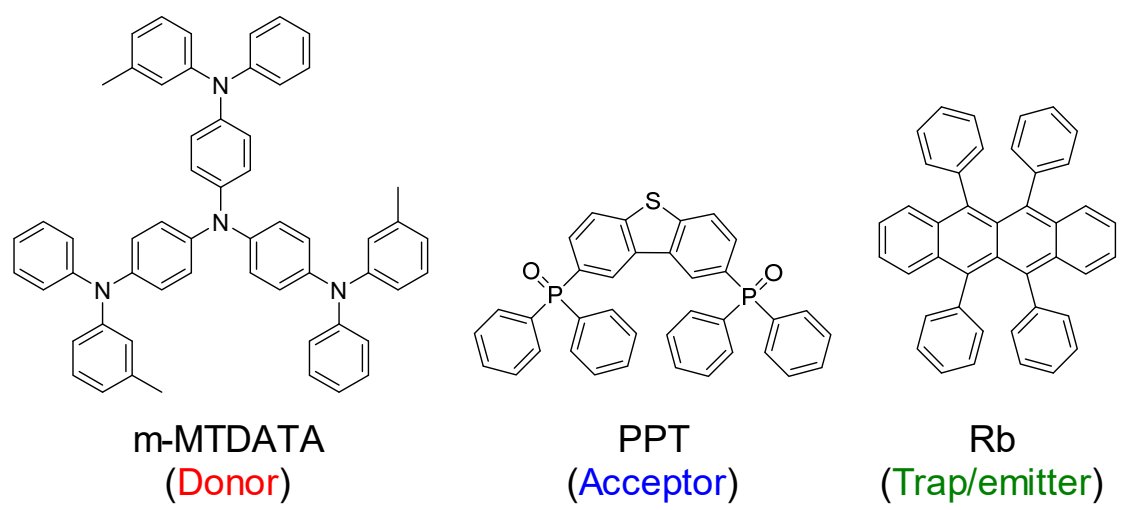

b

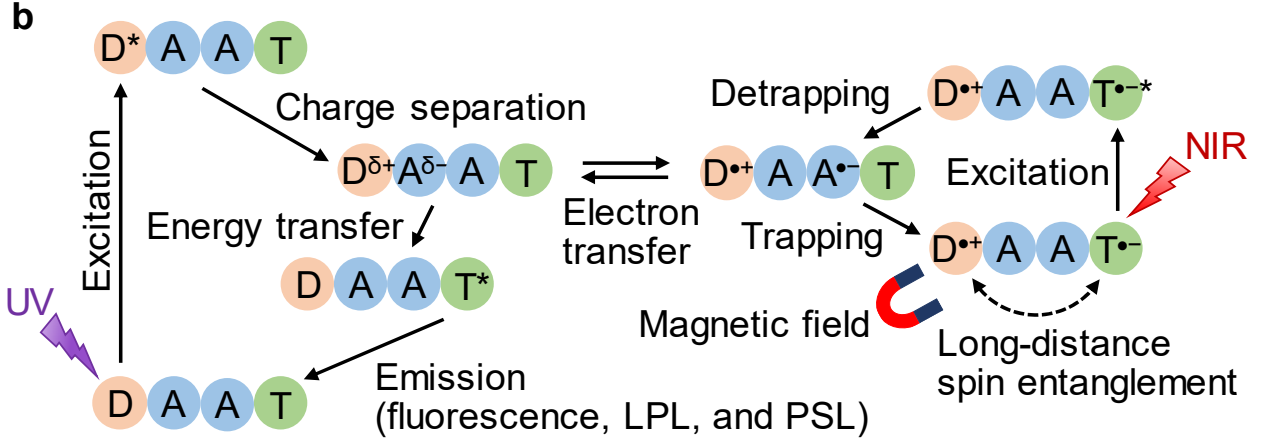

Fig. 1. Schematic illustration of organic PSL systems. a, Molecular structures of mMTDATA, PPT, and Rb used as electron donor, acceptor, and trap/emitter molecules, respectively. b, Energy diagram of organic PSL system. During UV excitation, an electron is transferred from the excited state of donor (D) to acceptor (A) to form the CT excited state or exciplex $\left(\mathrm{D}^{\delta+} \mathrm{A}^{\delta-}\right)$. The electrons in the film diffuse between $\mathrm{A}$ molecules and are partly captured by trap/emitter $(\mathrm{T})$ molecules, forming their radical anions $\left(\mathrm{T}^{\bullet^{-}}\right)$. The excited state of $\mathrm{T}^{\bullet^{-}}\left(\mathrm{T}^{\bullet^{-*}}\right)$ formed by NIR stimulation causes electron transfer to A, followed by reencountering of $\mathrm{A}^{\bullet-}$ with $\mathrm{D}^{\bullet+}$. The excited state of $\mathrm{T}\left(\mathrm{T}^{*}\right)$ is then formed via FRET from the regenerated $\mathrm{CT}$ state, resulting in visible PSL in addition to LPL. The luminescence from $\mathrm{T}^{*}$ is also modulated by external magnetic fields through long-distance spin entanglement between singlet and triplet states of the $\mathrm{D}^{\bullet+} \ldots \mathrm{T}^{\bullet-}$ pair. 


\section{Organic PSL characteristics}

Optical write-in and read-out processes based on organic PSL are schematically illustrated in Fig. 2a. First, an organic film is exposed to UV light for approximately one minute to write in information. The irradiated film is kept in the dark for a defined time and then exposed to NIR light for reading out the stored information as visible PSL. Figure $2 \mathrm{~b}$ displays a photograph of the m-MTDATA/PPT/Rb film prepared by reported procedures in an argon-filled glove box. ${ }^{15}$ After stopping UV irradiation for one minute, orange LPL was clearly seen from the film, except for from the masked region, and gradually weakened over time. Surprisingly, NIR light irradiation of the film dramatically enhanced this orange emission to the point of being visible to the naked eye after keeping the film in the dark at room temperature for one day after stopping UV irradiation and with a commercial digital camera one week after UV irradiation (Fig. 2c). The irradiated triangular area seemed to have been retained to ensure long-term storage ability, although emission was seen in cracks within the masked region probably due to light scattering or waveguiding inside the film. In the absence of $\mathrm{Rb}$, such a significant enhancement was not observed.

Luminescence properties of the films were examined by using an inverted fluorescence microscope (Extended Data Fig. 2a). To avoid the effects of structural heterogeneities such as cracks, uniform regions were carefully selected and measured (Extended Data Figs. 2b and c). Typical time profiles of LPL after stopping UV irradiation are shown in Fig. 2d. The LPL intensity suddenly increased more than 15 times upon NIR irradiation (see the arrow) without apparent changes in the spectral shape (Extended Data Fig. 3a). This observation is indicative of PSL, which could be repeated for 10 write-in/read-out cycles for the same sample with an $\sim 10 \%$ loss in initial intensity (Extended Data Fig. 3b).

To confirm the existence of $\mathrm{Rb}^{\bullet-}$, we measured optical absorption spectra for the $\mathrm{m}$ MTDATA/PPT/Rb ( $\mathrm{Rb}$ concentration of 0 or $1 \mathrm{~mol} \%$ ) films before and after UV irradiation, 
and then derived differential spectra. As shown in Fig. 2e and Extended Data Fig. 4, characteristic absorption bands of $\mathrm{Rb}^{\bullet-}$ were observed at approximately 800 and 1,000 $\mathrm{nm}^{21}$ only for the film containing $\mathrm{Rb}$, while both films exhibited a broad band from 900 to $1,400 \mathrm{~nm}$, which is analogous to the absorption spectrum of m-MTDATA radical cations (mMTDATA $\left.^{\bullet+}\right){ }^{22}$ The absorption decayed slowly over time after stopping UV irradiation (Extended Data Figs. 5a-c), indicating a gradual depletion of radical species accumulated in the film. The existence of $\mathrm{Rb}^{\bullet-}$ was further supported by electron spin resonance (ESR) spectral measurements (Extended Data Fig. 6). 

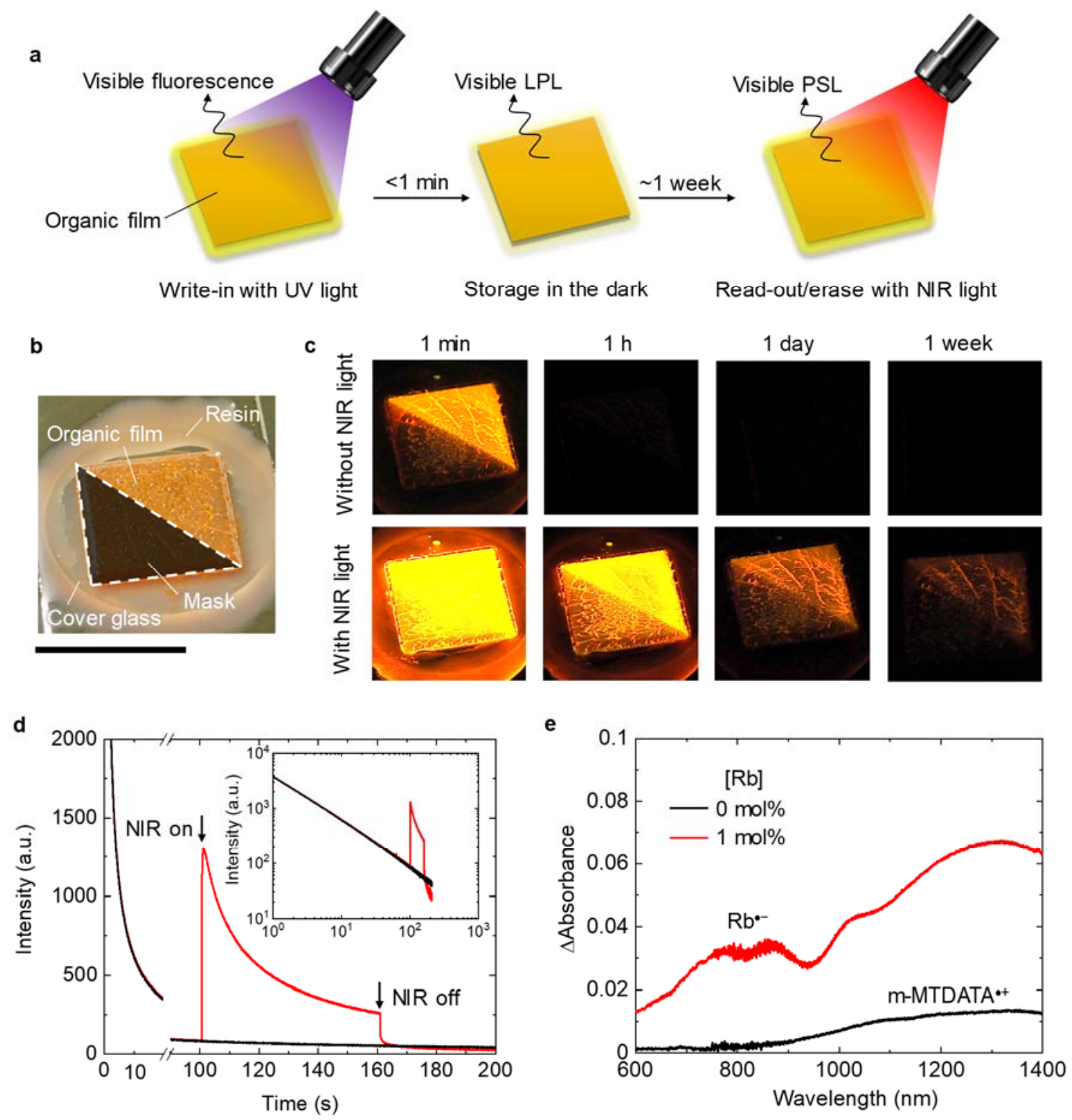

Fig. 2. Organic PSL from the m-MTDATA/PPT/Rb blend films. a, Write-in and read-out processes. b, Photograph of the m-MTDATA/PPT/Rb film. A part of the sample was masked during UV light irradiation. The scale bar is $10 \mathrm{~mm}$. c, Photographs of the samples without and with NIR light irradiation after stopping UV light irradiation. d, Emission decay profiles obtained for the m-MTDATA/PPT/Rb film with and without NIR irradiation (red and black lines, respectively). The inset shows the logarithmic plots of the decay profiles. The wavelengths of UV and NIR light were $365 \mathrm{~nm}$ and $800 \mathrm{~nm}$, respectively. e, Differential 
absorption spectra obtained for the m-MTDATA/PPT/Rb (0, 1 mol\%) films before and after 365-nm light irradiation.

To verify the origin of PSL, an action spectrum, where intensity changes upon NIR stimulation are plotted as a function of excitation wavelength, was measured. As shown in Extended Data Fig. 5d, the intensity change strongly depends on the excitation wavelength. For example, the intensity change at $850 \mathrm{~nm}$ is almost seven-fold greater than that at 1,000 nm, which corresponds to a photon energy $(\sim 1.24 \mathrm{eV})$ greater than the energy difference $(\sim 0.8 \mathrm{eV})$ between the LUMO levels of PPT and Rb (Extended Data Fig. 1). The obtained spectral shape roughly matches the absorption spectrum of $\mathrm{Rb}^{\bullet-}$, thus suggesting the importance of spectral matching between stimulation light and electronic absorption of radical anions of trap/emitter molecules. The electron transfer from the excited radical anions (e.g., perylene diimide radical anion) has been characterized by time-resolved spectroscopies and utilized for photoredox reactions such as reductive dehalogenation of aryl halides in spite of their short lifetimes on the pico- to nanosecond time scale. ${ }^{23-26}$ The high extinction coefficient of $\mathrm{Rb}^{\bullet-}\left(\varepsilon \approx 2 \times 10^{4} \mathrm{M}^{-1}\right.$ $\mathrm{cm}^{-1}$ at $\left.\sim 800 \mathrm{~nm}\right)^{21}$ is thus preferable for NIR stimulation. The intensity enhancement of the film without trap/emitter molecules was very low, which is consistent with the fact that negligible absorption was observed in Fig. $2 \mathrm{e}$, possibly due to a small $\varepsilon$ of $\mathrm{PPT}^{{ }^{--}}$. It can be concluded that the trap/emitter molecules in the film capture some of the electrons and release them as visible PSL by NIR excitation of their radical anion state (Fig. 1b).

\section{Multicolor Organic PSL}

The proposed scheme is applicable for color tuning with different trap/emitter molecules (2,5,8,11-tetra-tert-butylperylene (TBPe), 9,10-bis[ $N, N$-di-( $p$-tolyl)-amino $]$ anthracene (TTPA), 

(dicyanomethylene)-2-methyl-6-julolidyl-9-enyl-4H-pyran (DCM2)) whose LUMO levels are lower than that of PPT $(-2.2 \mathrm{eV})$. As demonstrated in Fig. 3 and Extended Data Fig. 3a, all the samples exhibited detectable PSL with different colors and spectra similar to their LPL without NIR irradiation. The m-MTDATA/PPT/TBRb film exhibited characteristics similar to the $\mathrm{Rb}$ sample because of the analogous structures of the trap/emitter molecules. DCM2 also showed a strong red PSL.

On the other hand, relatively weak NIR responses were observed for TBPe and TTPA. Since these molecules have LUMO energies lower than the others, we can exclude the possibility that the PSL simply originates from the thermal release of electrons from trap sites by NIR irradiation. In fact, significant PSL was observed for the m-MTDATA/PPT/Rb film even at $77 \mathrm{~K}$ (Extended Data Fig. 7). In the case of TBPe, the weak PSL may be due to inefficient excitation of radical anions since $\mathrm{TBPe}^{\bullet^{-}}$has very weak absorption bands in the NIR region. ${ }^{27}$ These results may also suggest that trapping of electrons at depths deeper than $0.5 \mathrm{eV}$ from the LUMO level of PPT could be necessary for intense PSL. Unfortunately, absorption spectra of radical anions of TTPA and DCM2 could not be obtained by spectroelectrochemistry due to simultaneous oxidation on a counter electrode and irreversibility in solution, respectively (Extended Data Fig. 4a). Further studies are thus required to establish the design principle of organic PSL systems. 


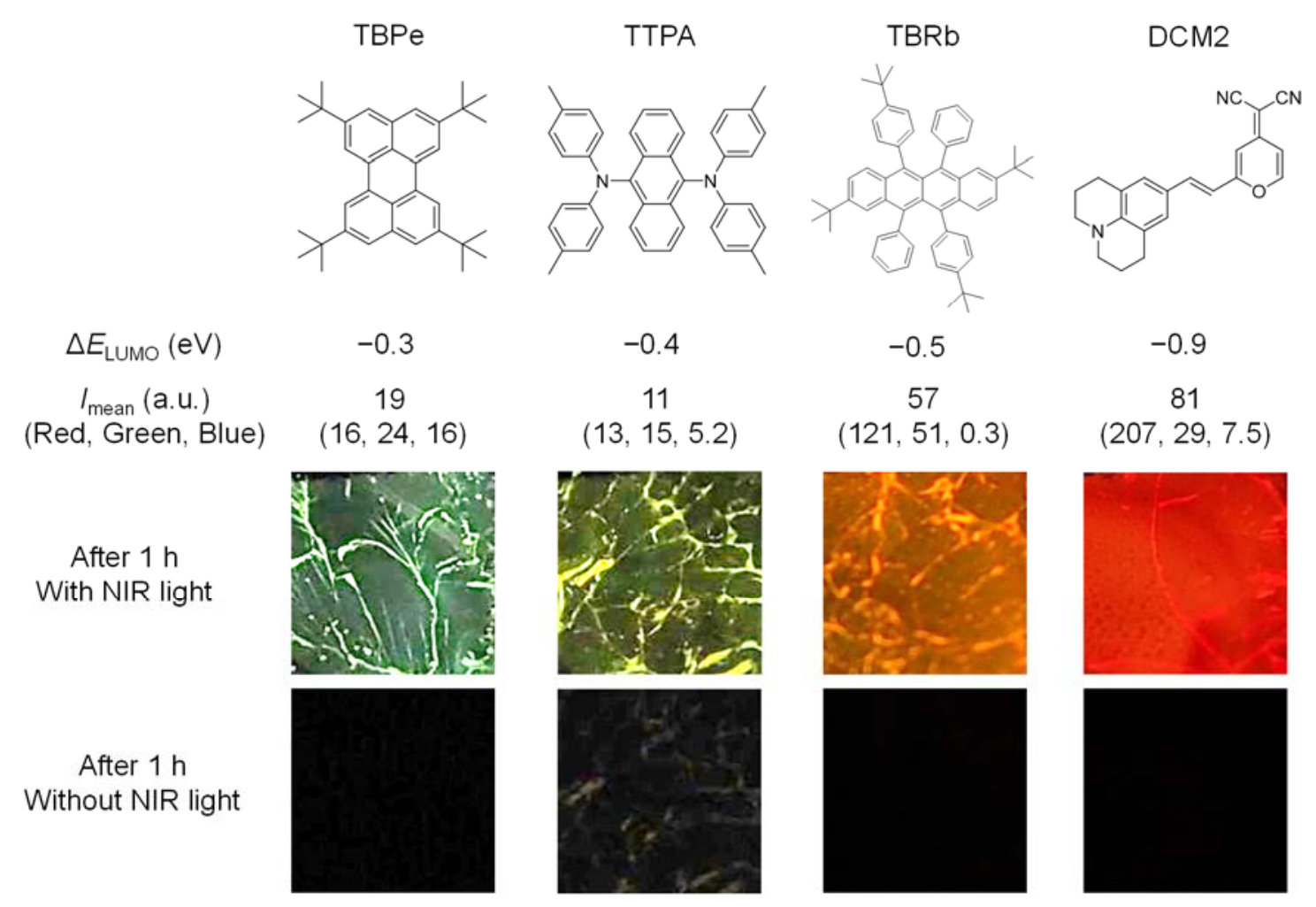

Fig. 3. Color tuning of PSL with different trap/emitter molecules. Molecular structures of trap/emitter molecules and corresponding photographs of the blend films containing different trap/emitter molecules $(1 \mathrm{~mol} \%)$ with and without NIR irradiation. The wavelengths of UV and NIR light were $365 \mathrm{~nm}$ and $800 \mathrm{~nm}$, respectively. The actual size of the films in the images is $5 \mathrm{~mm} \times 5 \mathrm{~mm}$. The difference of LUMO energy $\left(\Delta E_{\mathrm{LUMO}}\right)$ was calculated from $E_{\mathrm{LUMO}}$ (trap/emitter) $-E_{\text {LUMO }}(\mathrm{PPT})$ based on the reduction potentials of the materials (Extended Data Fig. 1). The mean intensity ( $\left.I_{\text {mean }}\right)$ of PSL was calculated from the red, green, and blue components of the color image taken by a digital camera. The exposure time was $10 \mathrm{~s}$. The brightness and contrast of images are different for each sample.

\section{Magnetic field effects}

To investigate the dynamics of the long-lived radical species in the blend films, magnetic field effects (MFEs) on LPL and PSL were examined by applying external magnetic fields $(B)$ with an electromagnet to the sample under the inverted fluorescence microscope. ${ }^{28}$ As demonstrated 
by previous studies, ${ }^{29-31}$ MFEs in organic light-emitting diodes (OLEDs) provide an underlying model for interconversion between singlet and triplet states that is highly related to device performance. Interestingly, as demonstrated in Fig. 4a and Extended Data Fig. 8, significant negative MFEs were observed for LPL and PSL, while no MFE was seen for the film without $\mathrm{Rb}$, implying that trap/emitter molecules play a crucial role.

The MFEs in the LPL process can be expressed as

$$
\chi_{\mathrm{LPL}}(B)=\frac{L P L(B, t)-L P L(0, t)}{L P L(0, t)}
$$

where $\operatorname{LPL}(B, t)$ and $L P L(0, t)$ represent the LPL intensity at time $t$ in the presence and absence of external magnetic field $(B)$, respectively. The $\chi_{\mathrm{LPL}}(B)$ values were plotted as a function of magnetic fields and were then fitted by the Lorentzian function (Fig. 4b). As summarized in the inset, the absolute values of saturated $\chi_{\mathrm{LPL}}(B)$ increased with increasing $\mathrm{Rb}$ concentration (Extended Data Fig. 8c), while the $B_{1 / 2}$ values (defined as the magnetic field at which the change in emission intensity reaches half of its saturation value) remained constant. A large deviation from the linearity for the $\mathrm{Rb}$ concentration of $5 \mathrm{~mol} \%$ is probably due to an aggregation of $\mathrm{Rb}$ molecules in the film. Meanwhile, as shown in Extended Data Fig. 9, positive MFEs were observed for fluorescence from the films both with and without Rb under UV irradiation. 

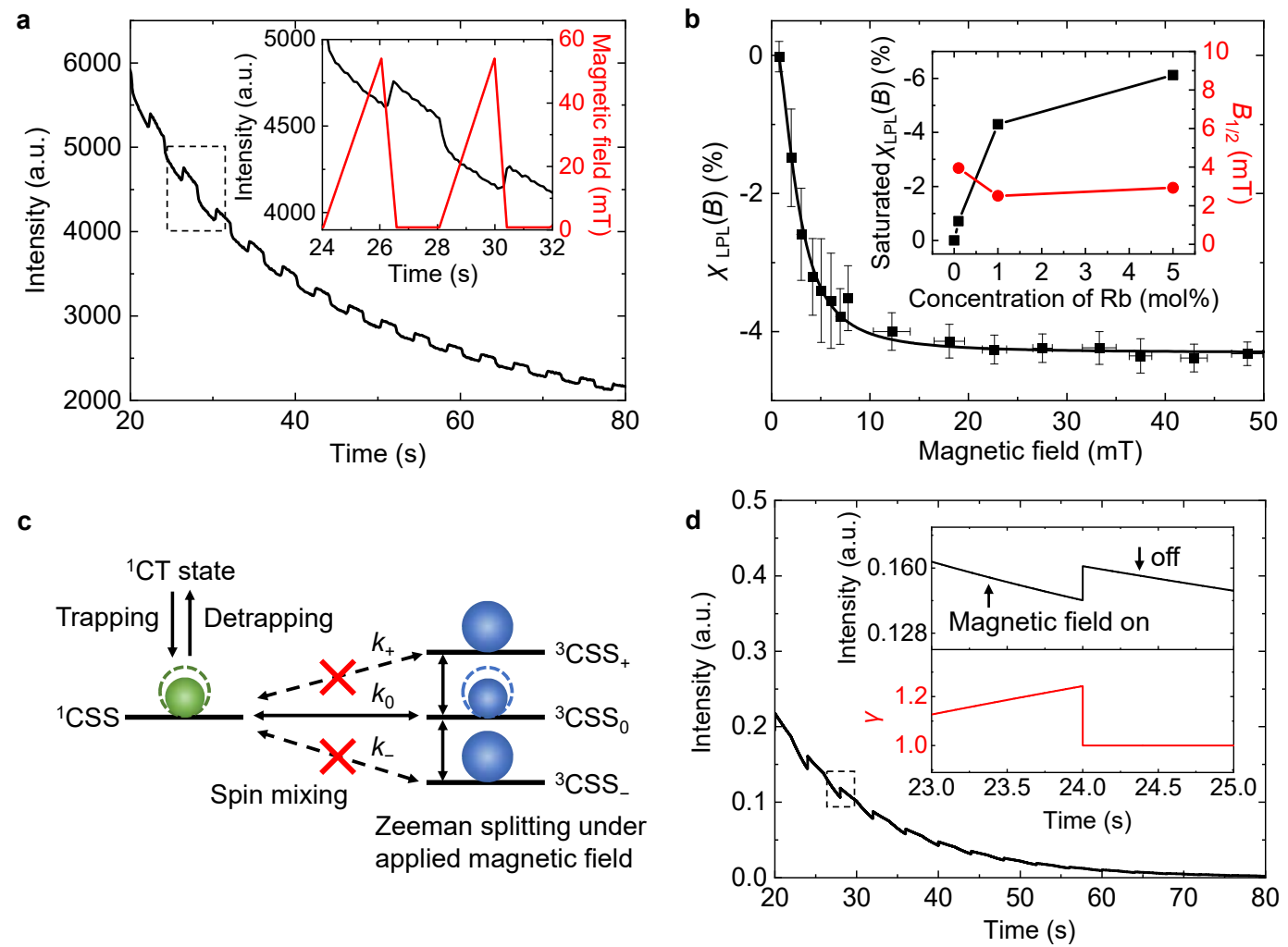

Fig. 4. Magnetic field effects. a, The response of LPL intensity to the external magnetic fields observed for the m-MTDATA/PPT/Rb film. The inset shows an enlarged view of the region contained within the dotted line and applied magnetic fields. $\mathbf{b}$, Plots of $\chi_{\operatorname{LPL}}(B)$ as a function of the external magnetic field $(B)$. The solid line shows the least-squares fit of data to the Lorentzian function. The inset indicates the dependence of the saturated $\chi_{\operatorname{LPL}}(B)$ and $B_{1 / 2}$ values on the Rb concentration. $\mathbf{c}$, A simplified model for magnetic field-induced population changes (circles in dotted lines) of singlet (green) and triplet (blue) charge-separated states (CSSs). d, The simulation of MFEs in the LPL process. The inset shows the temporal changes of the emission intensity (upper) and the population ratio $\gamma$ of ${ }^{1} \mathrm{CSS}$ and ${ }^{3} \mathrm{CSS}_{ \pm}$(lower) at around 23 to $25 \mathrm{~s}$ (see square in dotted line).

According to the fact that curve shapes of positive and negative MFEs and their $B_{1 / 2}$ values are almost exactly the same (Fig. $4 \mathrm{~b}$ and Extended Data Figs. 8 and 9), similar 
interactions governing spin conversion may be involved. However, the population of the spin states is considered to be different from each other. In the fluorescence process, electron transfer from the excited donor molecules generated by UV irradiation is the main pathway for formation of emissive singlet CT $\left({ }^{1} \mathrm{CT}\right)$ excited states (Fig. 4c). Since the singlet chargeseparated state $\left({ }^{1} \mathrm{CSS}\right)$ remains more populated than the triplet state $\left({ }^{3} \mathrm{CSS}_{0, \pm}\right)$ under continuous excitation, Zeeman splitting of the triplet sublevels by magnetic fields would inhibit intersystem crossing (ISC) from ${ }^{1} \mathrm{CSS}$ to ${ }^{3} \mathrm{CSS}_{ \pm}$, resulting in the increase of fluorescence intensity. Such a positive MFE was seen for the so-called magnetophotoluminescence behavior in OLEDs, and can be interpreted in terms of hyperfine interaction and/or $\Delta g$ mechanisms. ${ }^{29,30}$ On the other hand, negative MFEs in the LPL and PSL processes cannot be explained by the inhibition of forward ISC.

In organic LPL materials, a number of CSSs are stored in the film under and after preexcitation (Extended Data Figs. 5a-c). The spin distribution progresses to a 1:3 equilibrium based on spin statistics when spin mixing effectively occurs within the lifetime of spincorrelated CSSs (Fig. 4c). In the presence of external magnetic fields of approximately $10 \mathrm{mT}$, Zeeman splitting suppresses the population flow from ${ }^{3} \mathrm{CSS}_{ \pm}$to ${ }^{1} \mathrm{CSS}$ via spin mixing, resulting in the decrease of LPL intensity. It has been reported that triplets accumulated inside the OLED devices possess long lifetimes while keeping their spin states, resulting in negative MFEs, ${ }^{32}$ which are quite similar to our case. As demonstrated in Fig. 4b and Extended Data Fig. 9c, increased $\mathrm{Rb}$ concentrations led to increases in the absolute values of $\chi_{\mathrm{LPL} / \mathrm{FL}}(B)$. This result implies that the trap/emitter molecules effectively trap the electrons to form long-distance CSSs with negligible exchange interactions (Fig. 1b). ${ }^{33,34}$ From the density $\left(1.34 \pm 0.1 \mathrm{~g} \mathrm{~cm}^{-3}\right.$ ) and molecular weight $\left(584.6 \mathrm{~mol} \mathrm{~g}^{-1}\right)$ of PPT, a mean distance between m-MTDATA and trap/emitter molecules of $1 \mathrm{~mol} \%$ can be roughly estimated to be approximately $3 \mathrm{~nm}$, which matches the range $(>1 \mathrm{~nm})$ expected from the MFE results, taking its distribution into 
consideration. In addition to the trap/emitter molecules, the long lifetime of CSSs may be partly attributed to inherent trap sites (including structural disorders and impurities that are difficult to remove) in the m-MTDATA/PPT film.

\section{Numerical simulations}

These hypotheses were tested by numerical simulations based on a reaction kinetic model (Extended Data Fig. 10a). The details of the procedures are described in Supplementary Information. The ${ }^{1} \mathrm{CSS}$ is produced by electron diffusion or electron transfer to the traps from the ${ }^{1} \mathrm{CT}$ excited state generated by light irradiation, spin conversion from ${ }^{3} \mathrm{CSS}$, and detrapping from ${ }^{1} \mathrm{CSS}$.

In the LPL process, based on the spin statistics, the populations of ${ }^{1} \mathrm{CSS}$ and ${ }^{3} \mathrm{CSS}_{ \pm}$are equal, while the concentration of both CSSs decreases due to charge recombination into the CT states. Since spin mixing is an equilibrium process, the population ratio of ${ }^{1} \mathrm{CSS}$ and ${ }^{3} \mathrm{CSS}_{ \pm}$ depends on the rate of outflow to ${ }^{1} \mathrm{CT}$. Here, we briefly express the spin population ratio $(\gamma)$ as $\gamma={ }^{3} \mathrm{CSS}_{ \pm} /{ }^{1} \mathrm{CSS}$, where $\gamma$ is less than 1 in the fluorescence process and is greater than 1 in the LPL process. In the absence of magnetic fields, the energy levels of ${ }^{1} \mathrm{CSS}$ and ${ }^{3} \mathrm{CSS}_{ \pm}$are almost the same. When the spin mixing rate $\left(k_{ \pm}\right)$between them is reduced by applying magnetic fields, the population of ${ }^{3} \mathrm{CSS}_{ \pm}$becomes larger than that of ${ }^{1} \mathrm{CSS}$ (i.e., $\gamma>1$ ) because the outflow of ${ }^{3} \mathrm{CSS}_{ \pm}$must go through only ${ }^{3} \mathrm{CT}$. The decrease of the ${ }^{1} \mathrm{CSS}$ population leads to the population decrease of emissive ${ }^{1} \mathrm{CT}$. Thus the LPL intensity decreases, resulting in negative MFEs.

As depicted in Fig. 4d, when in corresponding with the removal of the applied magnetic field of $B_{1 / 2}, k_{ \pm}$was increased from $0 \mathrm{~s}^{-1}$ to $1.6 \times 10^{8} \mathrm{~s}^{-1}$, the LPL intensity clearly increased because the population of emissive ${ }^{1} \mathrm{CT}$ state became greater than that of the non-emissive ${ }^{3} \mathrm{CT}$ state through opened spin mixing pathways from ${ }^{3} \mathrm{CSS}_{ \pm}$to ${ }^{1} \mathrm{CSS}$. A more detailed discussion about the underlying mechanisms of MFEs, which highlight the importance of electron 
trapping/detrapping, is given in Supplementary Information with additional simulation results (Extended Data Figs. 10b-e).

\section{Conclusions}

We have developed novel multicolor organic PSL systems that store a certain amount of charges as radical anions of trap/emitter molecules up to one week at room temperature. The combination of UV writing-in, NIR stimulation, and visible output provides the possibility for many applications including organic optical data storage, security ink, and luminescence probes for bioimaging. Furthermore, microscopic MFE analyses and numerical simulations verified that luminescence properties can be controlled by weak magnetic fields owing to the interconversion between singlet and triplet CSSs through trap-mediated long-distance spin entanglement. Combined with unique luminescence properties, manipulation of long-lived spin states in organic LPL/PSL materials will energize researchers in various fields such as molecular spintronics, which is a promising next-generation technology. ${ }^{35,36}$

\section{Methods}

Materials. m-MTDATA was obtained from Sigma-Aldrich. PPT was synthesized according to literature. ${ }^{37}$ The predicted density of PPT using Advanced Chemistry Development (ACD/Labs) software was taken from SciFinder for calculation of molecular distance. $\mathrm{Rb}$, TBPe, TTPA, TBRb, and DCM2 were obtained from Luminescence Technology Corp. All compounds were purified by sublimation and stored in an argon-filled glovebox.

Sample fabrication. The ternary blend films were prepared by reported procedures. ${ }^{15}$ Briefly, m-MTDATA (1 mol\%), PPT (98 mol\%), and trap/emitter molecules ( $1 \mathrm{~mol} \%$, unless otherwise noted) were dissolved in dichloromethane. Then, the solvent was removed under reduced pressure in the dark. The mixture was dried using three cycles of the freeze-pump method. In 
an argon-filled glove box, the dried mixture was placed on a glass plate and heated up to $250{ }^{\circ} \mathrm{C}$ for $10 \mathrm{~s}$. After melting, the substrate was rapidly cooled down to room temperature and encapsulated using a cover glass and UV-cured epoxy resin (Fig. 2b). For low temperature experiments, a quartz glass tube containing a mixture of PPT (98 mol\%), m-MTDATA (1 mol\%), and $\mathrm{Rb}(1 \mathrm{~mol} \%)$ was evacuated under vacuum at room temperature and then heated up to $250{ }^{\circ} \mathrm{C}$ to melt the mixture. After cooling down to room temperature, the sample tube was inserted into a transparent glass Dewar vessel. The vessel was filled with liquid nitrogen for emission measurements at $77 \mathrm{~K}$.

Characterizations. To observe PSL, the sample was excited by monochromatic NIR light (e.g., $800 \mathrm{~nm}, 12 \mathrm{~mW} \mathrm{~cm}^{-2}$ at the sample) emitted from a Xe lamp (Asahi Spectra, MAX-303) with a bandpass filter, after stopping UV light irradiation $\left(365 \mathrm{~nm}, 35 \mathrm{~mW} \mathrm{~cm}^{-2}\right.$ at the sample) using an LED light source (Thorlabs, M365LP1). Before repeated experiments, the sample was exposed to intense NIR light $(750-1050 \mathrm{~nm})$ from the Xe lamp for 10 min to remove as many long-lived trapped electrons as possible. The optical absorption spectra were obtained using UV-vis-NIR spectrophotometer (JASCO, V-770). The transient emission decay profile was obtained using a streak camera system (C10910). The sample was excited by a 355-nm pulse laser (PL2210, Ekspla) at $10 \mathrm{~Hz}$. The cyclic voltammetry (CV) was carried out using an electrochemical analyzer (BAS, Model 610E). The measurements were performed in dried and oxygen-free dichloromethane $\left(\mathrm{CH}_{2} \mathrm{Cl}_{2}\right)$ or $N, N$-dimethylformamide (DMF) using $0.1 \mathrm{M}$ tetrabutylammonium hexafluorophosphate $\left(\mathrm{TBAPF}_{6}\right)$ as a supporting electrolyte. A platinum fiber was used as a working electrode, glassy carbon as a counter electrode, and $\mathrm{Ag} / \mathrm{Ag}^{+}$as a reference electrode. Redox potentials were referenced against ferrocene/ferrocenium $\left(\mathrm{Fc} / \mathrm{Fc}^{+}\right)$. The highest occupied molecular orbital (HOMO) and LUMO energy levels were calculated according to the equations of $E_{\mathrm{HOMO}}$ or $\mathrm{LUMO}=-E_{\text {redox }}\left(\mathrm{vs} . \mathrm{Fc} / \mathrm{Fc}^{+}\right)-4.8 \mathrm{eV} \cdot{ }^{38}$ The absorption spectra of radical anion or radical cation of the materials were obtained by UV-vis-NIR 
spectrophotometer (Shimadzu, UV-3600 Plus). Samples in dried and oxygen-free $\mathrm{CH}_{2} \mathrm{Cl}_{2}$ or DMF with $0.1 \mathrm{M}$ TBAPF6 were oxidized or reduced by the platinum mesh electrode through the use of an electrochemical analyzer (BAS, Model 610E).

Fluorescence microscopy measurements and numerical simulations. The fluorescence microscopy measurements were performed on an inverted fluorescence microscope (Nikon, Ti-E). A 365-nm LED (Thorlabs, M365LP1; $0.85 \mathrm{~W} \mathrm{~cm}^{-2}$ at the sample) was used to excite the sample through an objective lens (CFI Plan Apo $\lambda 100 \times \mathrm{H}$, Nikon; NA (numerical aperture) $=$ 1.45). A $810-\mathrm{nm}$ LED light source (Thorlabs, M810L3; $810 \mathrm{~nm}, 12 \mathrm{~mW} \mathrm{~cm}^{-2}$ at the sample) was used for NIR stimulation. A Xe lamp (Asahi Spectra, MAX-303) and bandpass filters were used for NIR excitation with the same numbers of emitted photons to obtain the action spectrum. The NIR light was irradiated from above the sample. The emission from the sample was collected by the same objective lens, after which it was magnified by a $1.5 \times$ built-in magnification changer. It subsequently passed through a dichroic mirror (Semrock, FF697SDI01 or Di02-R405) and a shortpass filter (Semrock, FF02-694/SP-25) or a longpass filter (Semrock, BLP01-405R) to remove the undesired scattered light. The emission images were recorded with an electron-multiplying charge-coupled device camera (Roper Scientific, Evolve 512) using Micro-Manager (https://www.micro-manager.org/). For the spectroscopy, only the emission that passed through a long-pass filter (Semrock, BLP01-405R) and a slit entered the imaging spectrograph (SOL instruments, MS3504i) equipped with a CCD camera (Andor, DU416A-LDC-DD). Magnetic fields were applied using a custom-made electromagnet and calibrated with a gauss meter. Numerical simulations were performed using MATLAB and Simulink (The MathWorks). All experimental data were obtained at room temperature unless otherwise noted. 


\section{References}

1. Xu, J. \& Tanabe, S. Persistent luminescence instead of phosphorescence: History, mechanism, and perspective. J. Lumin. 205, 581-620 (2019).

2. Lakshmanan, A.R. Radiation-induced defects and photostimulated luminescence process in BaFBr:Eu ${ }^{2+}$. Phys. Status Solidi A 153, 3-27 (1996).

3. Rodriguez Burbano, D.C., Rodriguez, E.M., Dorenbos, P., Bettinelli, M. \& Capobianco, J.A. The near-IR photo-stimulated luminescence of $\mathrm{CaS}: \mathrm{Eu}^{2+} / \mathrm{Dy}^{3+}$ nanophosphors. $J$. Mater. Chem. C 2, 228-231 (2014).

4. Zhuang, Y., Katayama, Y., Ueda, J. \& Tanabe, S. A brief review on red to near-infrared persistent luminescence in transition-metal-activated phosphors. Opt. Mater. 36, 1907 1912 (2014).

5. Cho, J.H. et al. Printable ion-gel gate dielectrics for low-voltage polymer thin-film transistors on plastic. Nat. Mater. 7, 900-906 (2008).

6. Yan, H. et al. A high-mobility electron-transporting polymer for printed transistors. Nature 457, 679-686 (2009).

7. Sekitani, T., Zschieschang, U., Klauk, H. \& Someya, T. Flexible organic transistors and circuits with extreme bending stability. Nat. Mater. 9, 1015-1022 (2010).

8. Kaltenbrunner, M. et al. An ultra-lightweight design for imperceptible plastic electronics. Nature 499, 458-463 (2013).

9. Kato, M., Ito, H., Hasegawa, M. \& Ishii, K. Soft Crystals: Flexible Response Systems with High Structural Order. Chem. - Eur. J. 25, 5105-5112 (2019).

10. Irie, M., Fukaminato, T., Matsuda, K. \& Kobatake, S. Photochromism of Diarylethene Molecules and Crystals: Memories, Switches, and Actuators. Chem. Rev. 114, 1217412277 (2014).

11. Hosono, N. \& Kitagawa, S. Modular Design of Porous Soft Materials via Self- 
Organization of Metal-Organic Cages. Acc. Chem. Res. 51, 2437-2446 (2018).

12. Green, M.A., Ho-Baillie, A. \& Snaith, H.J. The emergence of perovskite solar cells. Nat. Photonics 8, 506-514 (2014).

13. Xing, G. et al. Low-Temperature Solution-Processed Wavelength-Tunable Perovskites for Lasing. Nat. Mater. 13, 476-480 (2014).

14. Kabe, R. \& Adachi, C. Organic long persistent luminescence. Nature 550, 384-387 (2017).

15. Jinnai, K., Kabe, R. \& Adachi, C. Wide-Range Tuning and Enhancement of Organic Long-Persistent Luminescence Using Emitter Dopants. Adv. Mater. 30, e1800365 (2018).

16. Lin, Z., Kabe, R., Nishimura, N., Jinnai, K. \& Adachi, C. Organic Long-Persistent Luminescence from a Flexible and Transparent Doped Polymer. Adv. Mater. 30, 1803713 (2018).

17. Lin, Z., Kabe, R., Wang, K. \& Adachi, C. Influence of energy gap between chargetransfer and locally excited states on organic long persistence luminescence. Nat. Commun. 11, 191 (2020).

18. Nishimura, N., Lin, Z., Jinnai, K., Kabe, R. \& Adachi, C. Many Exciplex Systems Exhibit Organic Long-Persistent Luminescence. Adv. Funct. Mater. 30, 2000795 (2020).

19. Alam, P. et al. Two Are Better Than One: A Design Principle for Ultralong-Persistent Luminescence of Pure Organics. Adv. Mater. 32, 2001026 (2020).

20. Jinnai, K., Nishimura, N., Kabe, R. \& Adachi, C. Fabrication-method Independence of Organic Long-persistent Luminescence Performance. Chem. Lett. 48, 270-273 (2019).

21. Saeki, A., Seki, S., Takenobu, T., Iwasa, Y. \& Tagawa, S. Mobility and dynamics of charge carriers in rubrene single crystals studied by flash-photolysis microwave conductivity and optical spectroscopy. Adv. Mater. 20, 920-923 (2008). 
22. Matsushima, T. et al. Interfacial charge transfer and charge generation in organic electronic devices. Org. Electron. 12, 520-528 (2011).

23. Gosztola, D., Niemczyk, M.P., Svec, W., Lukas, A.S. \& Wasielewski, M.R. Excited Doublet States of Electrochemically Generated Aromatic Imide and Diimide Radical Anions. J. Phys. Chem. A 104, 6545-6551 (2000).

24. Fujitsuka, M. \& Majima, T. Reaction dynamics of excited radical ions revealed by femtosecond laser flash photolysis. J. Photochem. Photobiol., C 35, 25-37 (2018).

25. Ghosh, I., Ghosh, T., Bardagi, J.I. \& Koenig, B. Reduction of aryl halides by consecutive visible light-induced electron transfer processes. Science 346, 725-728 (2014).

26. Zeman, C.J., Kim, S., Zhang, F. \& Schanze, K.S. Direct Observation of the Reduction of Aryl Halides by a Photoexcited Perylene Diimide Radical Anion. J. Am. Chem. Soc. 142, 2204-2207 (2020).

27. Karabunarliev, S., Gherghel, L., Koch, K.-H. \& Baumgarten, M. Structure and optical absorption of oligorylenes upon doping. Chem. Phys. 189, 53-65 (1994).

28. Sakurai, M., Kobori, Y. \& Tachikawa, T. Structural Dynamics of Lipid Bilayer Membranes Explored by Magnetic Field Effect Based Fluorescence Microscopy. $J$. Phys. Chem. B 123, 10896-10902 (2019).

29. Baniya, S. et al. Magnetic field effect in organic light-emitting diodes based on electron donor-acceptor exciplex chromophores doped with fluorescent emitters. Adv. Funct. Mater. 26, 6930-6937 (2016).

30. Basel, T. et al. Magnetic Field Enhancement of Organic Light-Emitting Diodes Based on Electron Donor-Acceptor Exciplex. Adv. Electron. Mater. 2, 1500248 (2016).

31. Crooker, S.A. et al. Spectrally resolved hyperfine interactions between polaron and nuclear spins in organic light emitting diodes: Magneto-electroluminescence studies. 
Appl. Phys. Lett. 105, 153304 (2014).

32. Peng, Q. et al. Evidence of the Reverse Intersystem Crossing in Intra-Molecular Charge-Transfer Fluorescence-Based Organic Light-Emitting Devices Through Magneto-Electroluminescence Measurements. Adv. Opt. Mater. 1, 362-366 (2013).

33. Zhang, C. et al. Magnetic Field Effects in Hybrid Perovskite Devices. Nat. Phys. 11, 427-434 (2015).

34. Woodward, J.R. Radical pairs in solution. Prog. React. Kinet. Mech. 27, 165-207 (2002).

35. Wolf, S.A. et al. Spintronics: A spin-based electronics vision for the future. Science 294, 1488-1495 (2001).

36. Ratera, I. \& Veciana, J. Playing with organic radicals as building blocks for functional molecular materials. Chem. Soc. Rev. 41, 303-349 (2012).

37. Fan, C. et al. Dibenzothiophene-Based Phosphine Oxide Host and ElectronTransporting Materials for Efficient Blue Thermally Activated Delayed Fluorescence Diodes through Compatibility Optimization. Chem. Mater. 27, 5131-5140 (2015).

38. Pommerehne, J. et al. Efficient two layer LEDs on a polymer blend basis. Adv. Mater. 7, 551-554 (1995).

\section{Data availability}

The data associated with the reported findings are available in the manuscript or the Supplementary Information. Other related data are available from the corresponding authors upon request.

\section{Code availability}

The analysis codes that support the findings of the study are available from the corresponding authors on reasonable request. 


\section{Acknowledgements}

We thank W. J. Potscavage Jr for assistance with manuscript preparation. This work was partially supported by JSPS KAKENHI Grant Numbers JP18H04517, JP18H04517, JP20H04673, JP18H02049, JP18H04522, the Japan Science and Technology Agency (JST), ERATO, Adachi Molecular Exciton Engineering Project, under JST ERATO Grant Number JPMJER1305, Japan, the International Institute for Carbon Neutral Energy Research (WPII2CNER) sponsored by the Ministry of Education, Culture, Sports, Science and Technology (MEXT), and others.

\section{Author contributions}

R.K. and T.T. conceived the project. M.S., R.K., and T.T. prepared the samples and obtained the experimental data. M.F. and Y.K. performed ESR measurements and Gaussian calculations. R.K., Z.L, and K.J. performed electrochemical and spectroelectrochemical measurements. K.J performed transient emission decay measurements. M.S. and T.T. performed numerical simulations. C.A. partly guided the project. M.S., R.K., and T.T. wrote the manuscript. All the authors discussed the results and commented on the manuscript.

Competing interests. The authors declare no competing interests. 


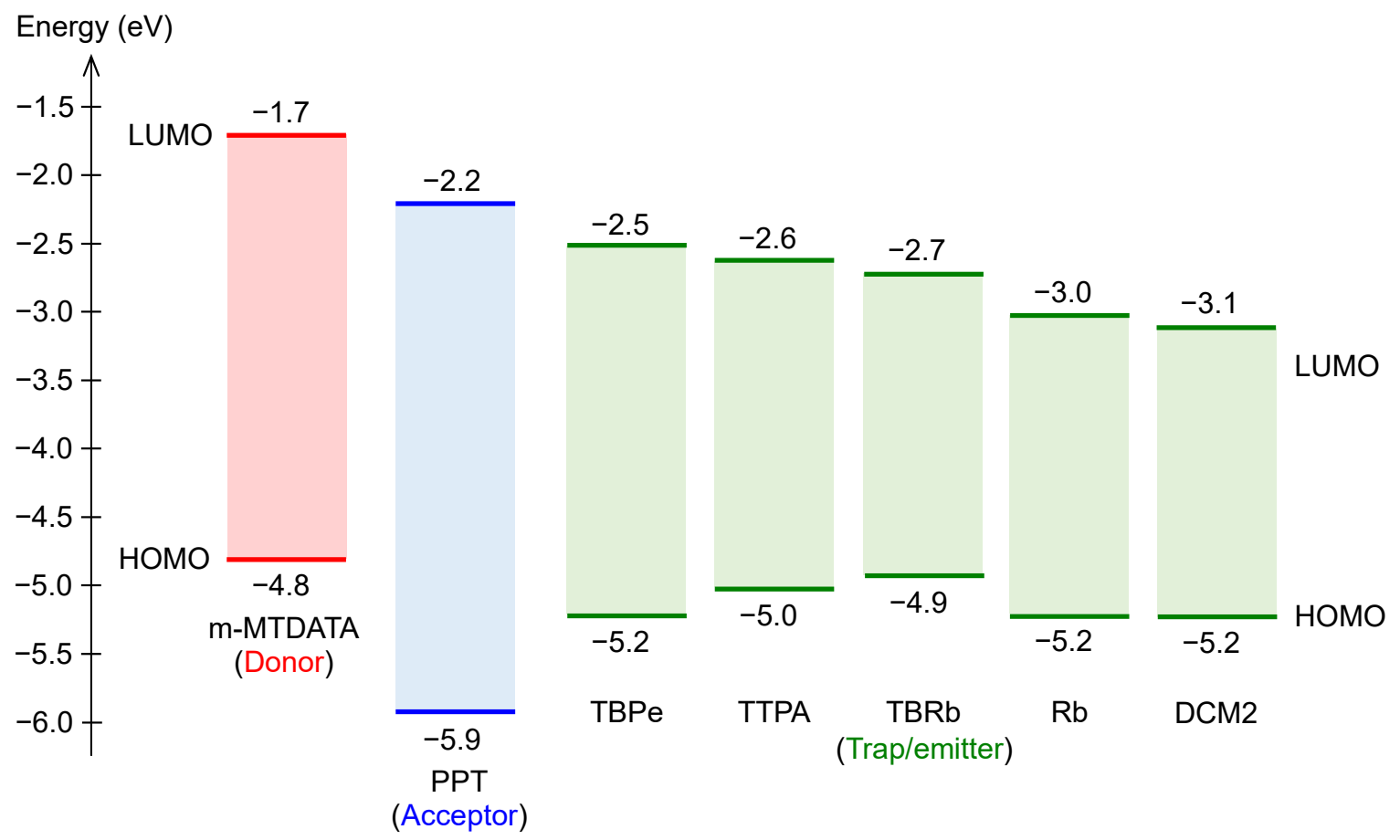

Extended Data Fig. 1. HOMO and LUMO energy levels. The HOMO level of m-MTDATA and LUMO levels of PPT and trap/emitter molecules were calculated from redox peaks of cyclic voltammograms. The energy gaps between HOMO and LUMO levels were calculated from the onsets of the absorption spectra observed for solution samples. 
a

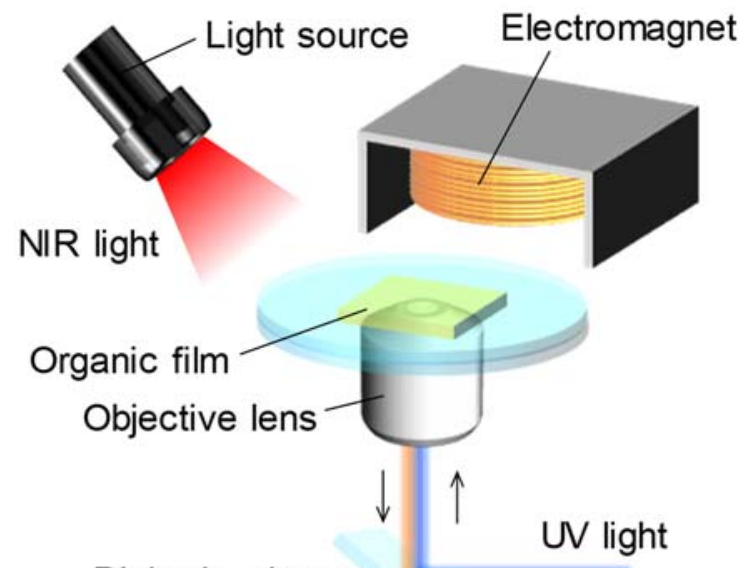

Dichroic mirror

Longpass or bandpass filter
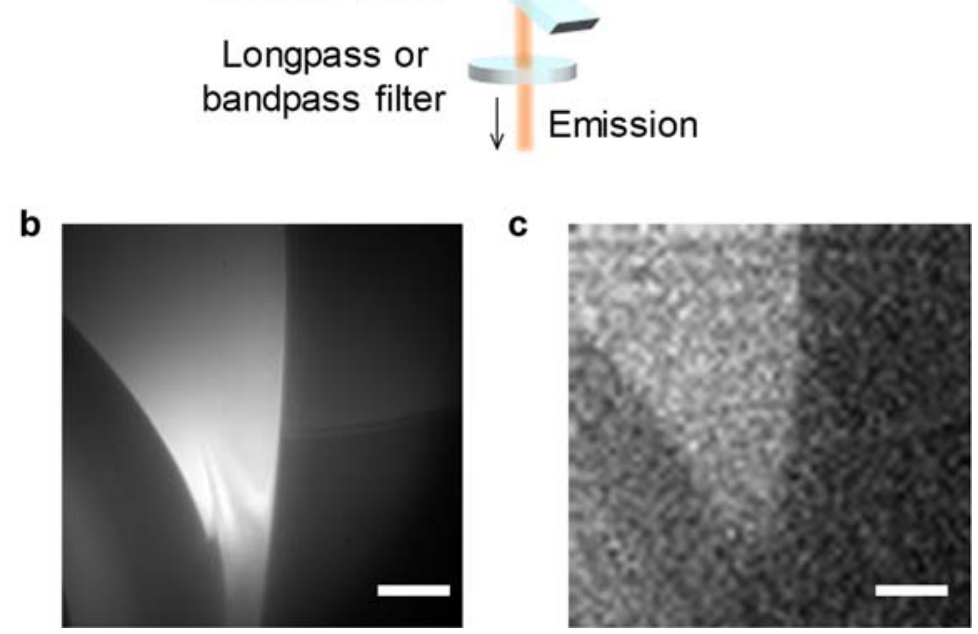

Extended Data Fig. 2. Fluorescence microscopy measurements. a, Experimental setup based on inverted fluorescence microscope. The NIR light and magnetic fields were applied from above the sample. b, Fluorescence image observed for the m-MTDATA/PPT/Rb film under 365-nm light irradiation. c, LPL image observed for the m-MTDATA/PPT/Rb film at 10 s after stopping 365-nm light irradiation. The scale bars are $10 \mu \mathrm{m}$. The uniformly emitting regions without apparent structural disorders were selected for analysis. 

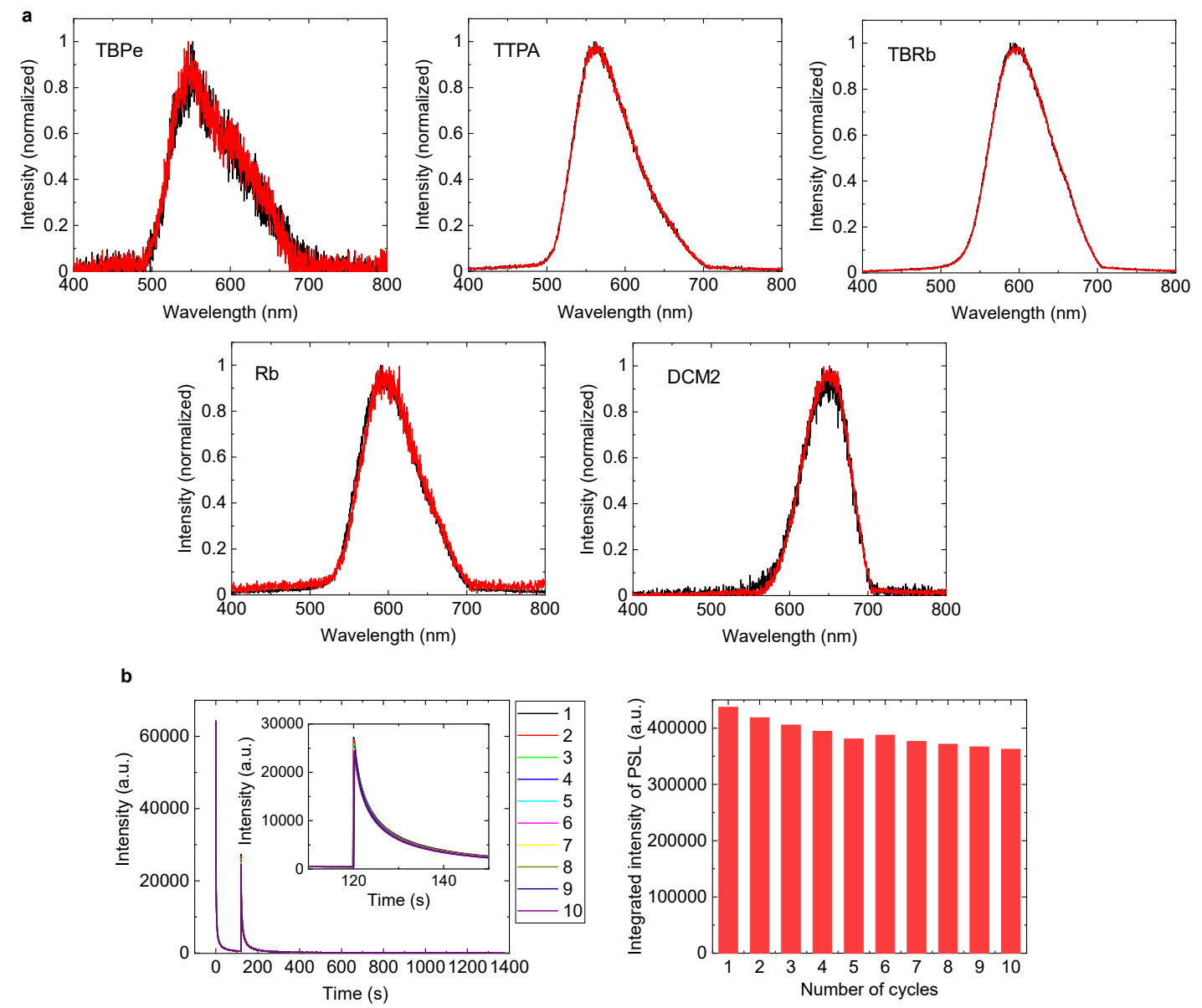

Extended Data Fig. 3. Emission characteristics of the films. a, Normalized emission spectra of the m-MTDATA/PPT films containing $1 \mathrm{~mol} \%$ trap/emitter molecules $(\mathrm{Rb}$, TBPe, TTPA, $\mathrm{TBR}$, or DCM2) without (black line) and with (red line) NIR irradiation. Prior to the measurements using fluorescence microscopy, the sample was excited by UV light to accumulate the charges. The wavelengths of UV and NIR light were $365 \mathrm{~nm}$ and $810 \mathrm{~nm}$, respectively. Note that the blue $(\sim 500 \mathrm{~nm})$ and red $(\sim 700 \mathrm{~nm})$ edges of the spectra were removed by the shortpass filter and dichroic mirror, respectively. $\mathbf{b}$, Time traces of emission intensity (left) and changes of PSL intensity integrated over a period of $20 \mathrm{~min}$ (right) obtained from 10 repeated experiments. The inset in the left panel shows the initial time profiles of PSL. First, the sample was exposed to 365-nm light for $60 \mathrm{~s}$. After being kept in the dark for 2 min, the sample was exposed to 800 -nm light for $20 \mathrm{~min}$, followed by a cooling in the dark for 10 min. This cycle was repeated 10 times. 
a

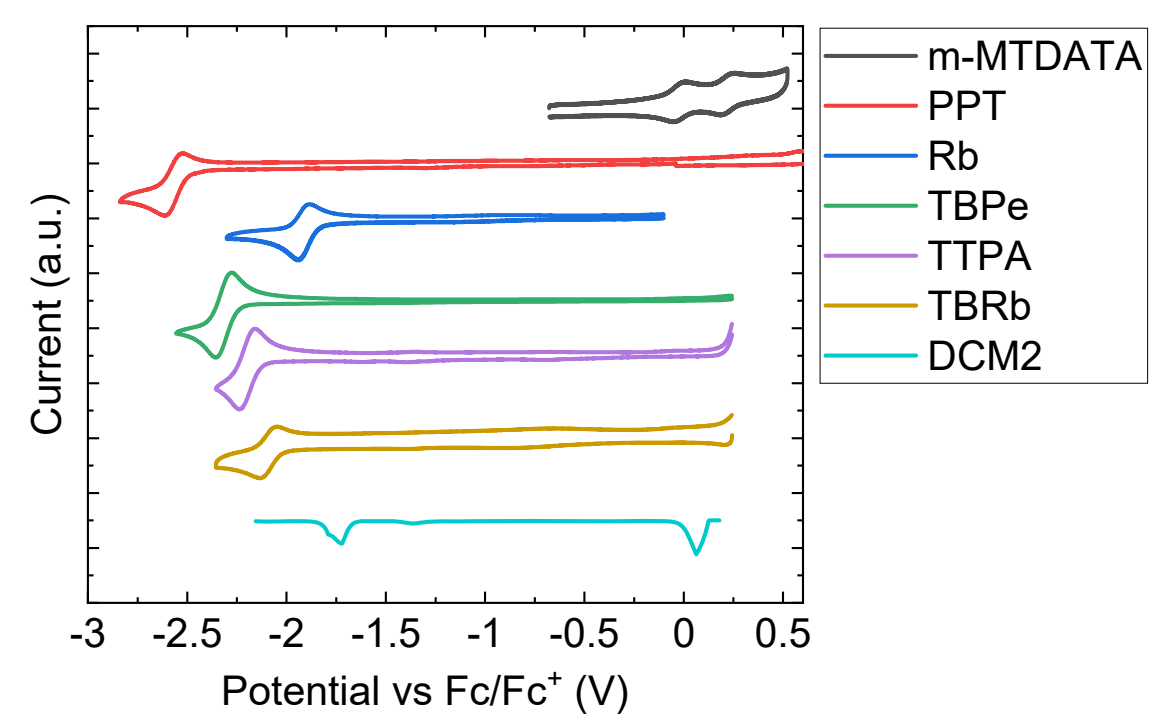

b

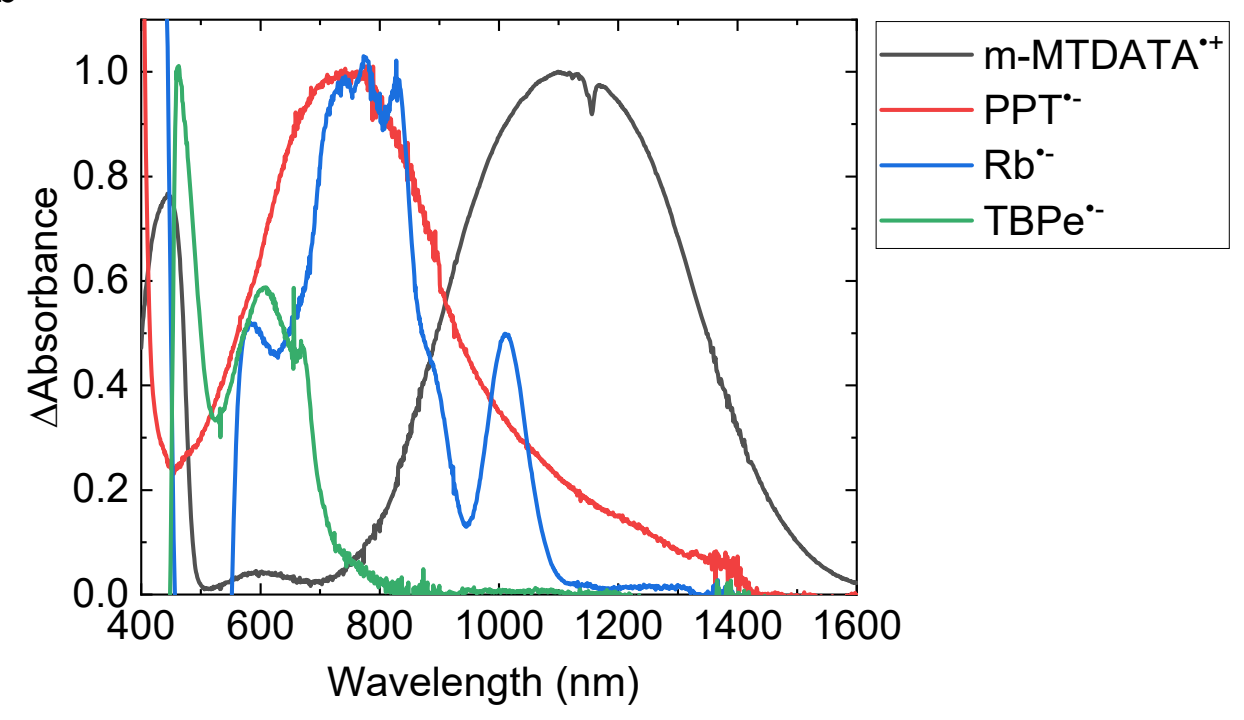

Extended Data Fig. 4. Electrochemical measurements. a, Cyclic voltammograms of organic compounds used in this study. $\mathbf{b}$, Absorption spectra of m-MTDATA radical cation, PPT radical anion, $\mathrm{Rb}$ radical anion, and TBPe radical anion obtained by difference absorption spectra of electrical oxidation or reduction. 

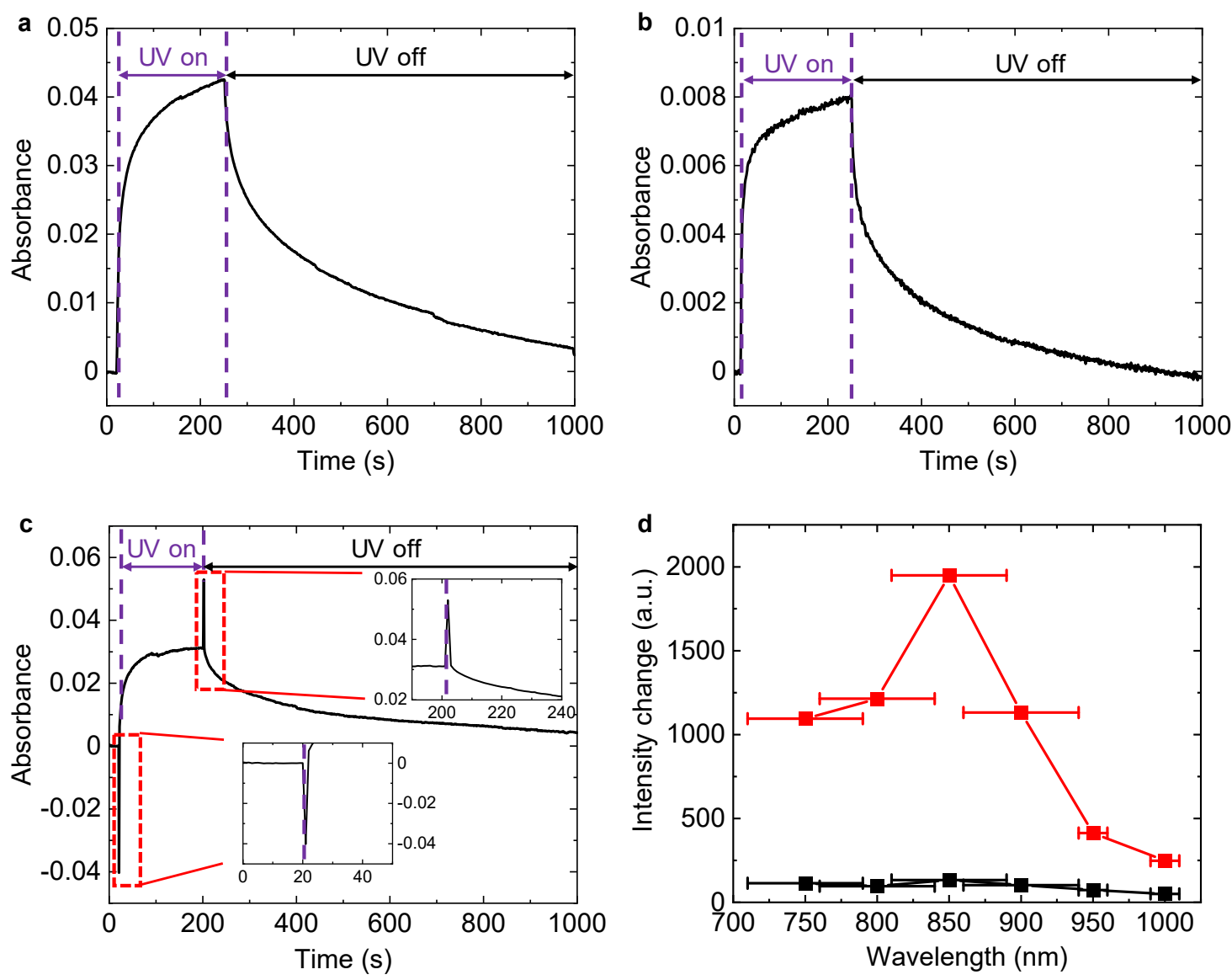

Extended Data Fig. 5. Observation of long-lived radicals. a, Time profile of differential absorbance observed at $1100 \mathrm{~nm}$ for the m-MTDATA/PPT/Rb film. b, Time profile of differential absorbance observed at $1100 \mathrm{~nm}$ for the m-MTDATA/PPT film. c, Time profile of differential absorbance observed at $740 \mathrm{~nm}$ for the m-MTDATA/ PPT/Rb film. The excitation wavelength was $365 \mathrm{~nm}$. d, Emission intensity changes at each excitation wavelength observed for the m-MTDATA/PPT (black) and m-MTDATA/PPT/Rb (red) films at $100 \mathrm{~s}$ after stopping 365-nm light irradiation. The error bars indicate the bandwidth (FWHM) of the bandpass filters (Thorlabs) used for monochromatic excitation with the same number of emitted photons. 

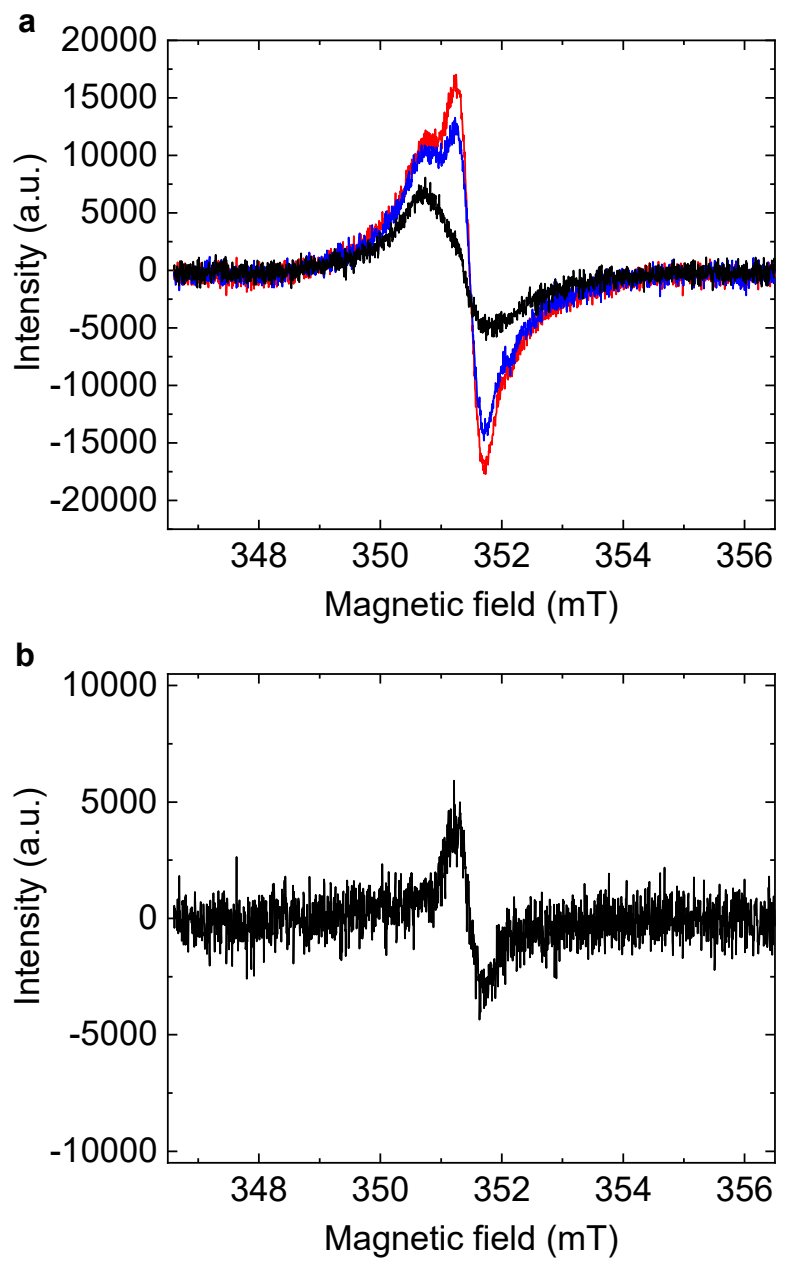

Extended Data Fig. 6. ESR measurements. a, ESR spectra of the m-MTDATA/PPT/Rb film under dark condition (black line), UV irradiation (red line), and UV and NIR irradiation (blue line). The wavelengths of UV and NIR light were $365 \mathrm{~nm}$ and $810 \mathrm{~nm}$, respectively. b, Differential ESR spectrum obtained by subtracting the blue line from the red line in panel a. 
a at $298 \mathrm{~K}$
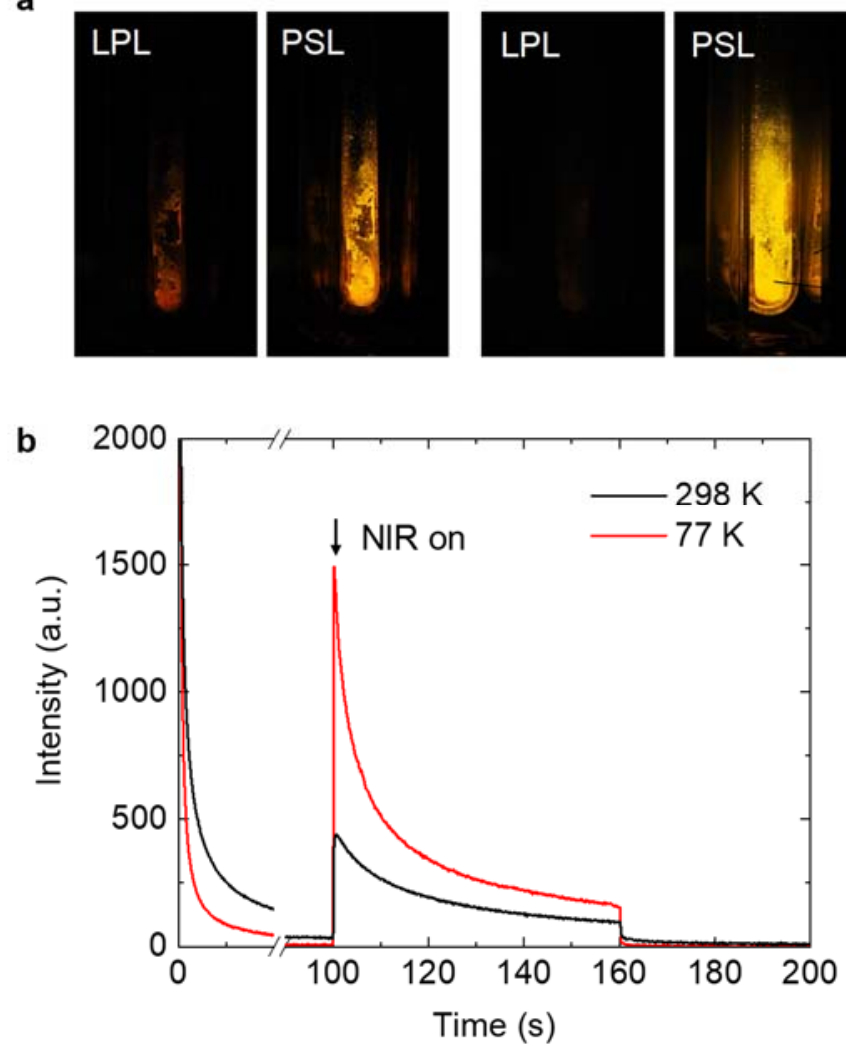

Extended Data Fig. 7. PSL at 77 K. a, Photographs showing LPL and PSL captured for the m-MTDATA/PPT/Rb amorphous solid at $298 \mathrm{~K}$ (left) and $77 \mathrm{~K}$ (right). The photographs of LPL were obtained after stopping UV light irradiation for $60 \mathrm{~s}$. The photographs of PSL were obtained under NIR light irradiation at $100 \mathrm{~s}$ after stopping UV light irradiation for $60 \mathrm{~s}$. The acquisition times were $10 \mathrm{~s}$. b, Emission decay profiles obtained for the m-MTDATA/PPT/Rb amorphous solid at $298 \mathrm{~K}$ (black) and $77 \mathrm{~K}$ (red) with and without NIR irradiation (see the arrow). The wavelengths of UV and NIR light were $365 \mathrm{~nm}$ and $850 \mathrm{~nm}$, respectively. The sample was placed just above the objective lens (Extended Data Fig. 2a) and exposed to UV or NIR light. The wavelengths of UV and NIR light were $365 \mathrm{~nm}$ and $850 \mathrm{~nm}$, respectively. 

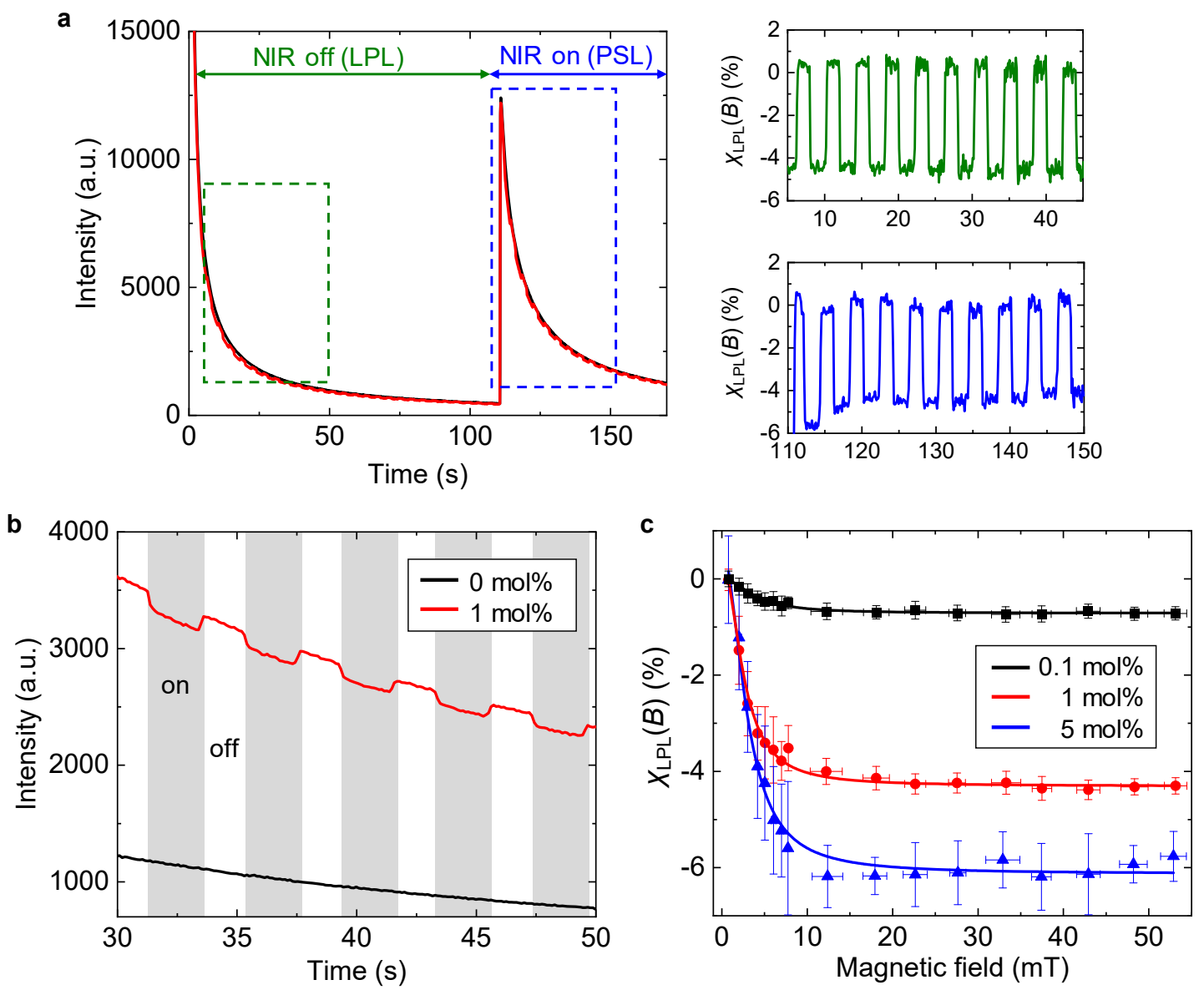

Extended Data Fig. 8 MFEs on LPL. a, MFEs on the LPL decay profiles of the mMTDATA/PPT/Rb (1 mol\%) film with and without NIR irradiation. The magnetic fields were modulated between 0.6 to $30 \mathrm{mT}$. The wavelengths of UV and NIR light was $365 \mathrm{~nm}$ and 800 $\mathrm{nm}$, respectively. $\chi_{\mathrm{LPL}}(B)$ values obtained without (upper right) and with (lower right) NIR irradiation. b, Responses of LPL intensity obtained for the films with (red line) and without (black line) $\mathrm{Rb}(1 \mathrm{~mol} \%)$ to the external magnetic field after stopping UV irradiation. The gray color indicates the application of a magnetic field. $\mathbf{c}$, Plots of $\chi \operatorname{LPL}(B)$ as a function of magnetic field obtained for the m-MTDATA $(1 \mathrm{~mol} \%) / \mathrm{PPT} / \mathrm{Rb}(0.1,1$, and $5 \mathrm{~mol} \%)$ films. The solid lines indicate the fitted data by the Lorentzian function. 

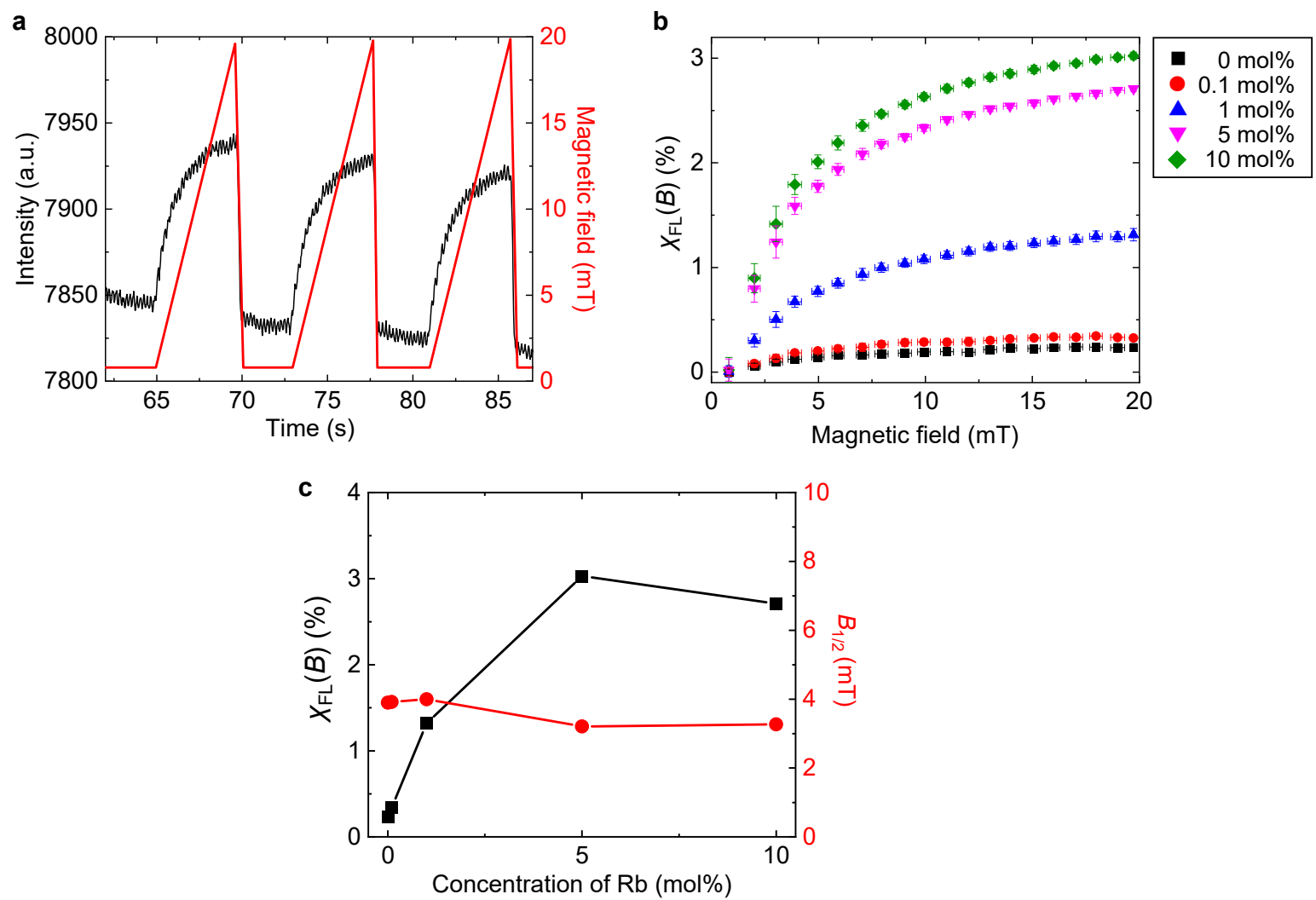

Extended Data Fig. 9. MFEs on fluorescence. a, Fluorescence intensity response of the mMTDATA/PPT/Rb film to the external magnetic fields under UV irradiation. The excitation wavelength was $365 \mathrm{~nm}$. b, Plots of $\chi_{\mathrm{FL}}(B)$ values as a function of magnetic field observed for the m-MTDATA/PPT/Rb $(0,0.1,1,5$, and $10 \mathrm{~mol} \%)$ films. c, Dependences of the $\chi_{\mathrm{FL}}$ and $B_{1 / 2}$ values on the $\mathrm{Rb}$ concentration. 
a
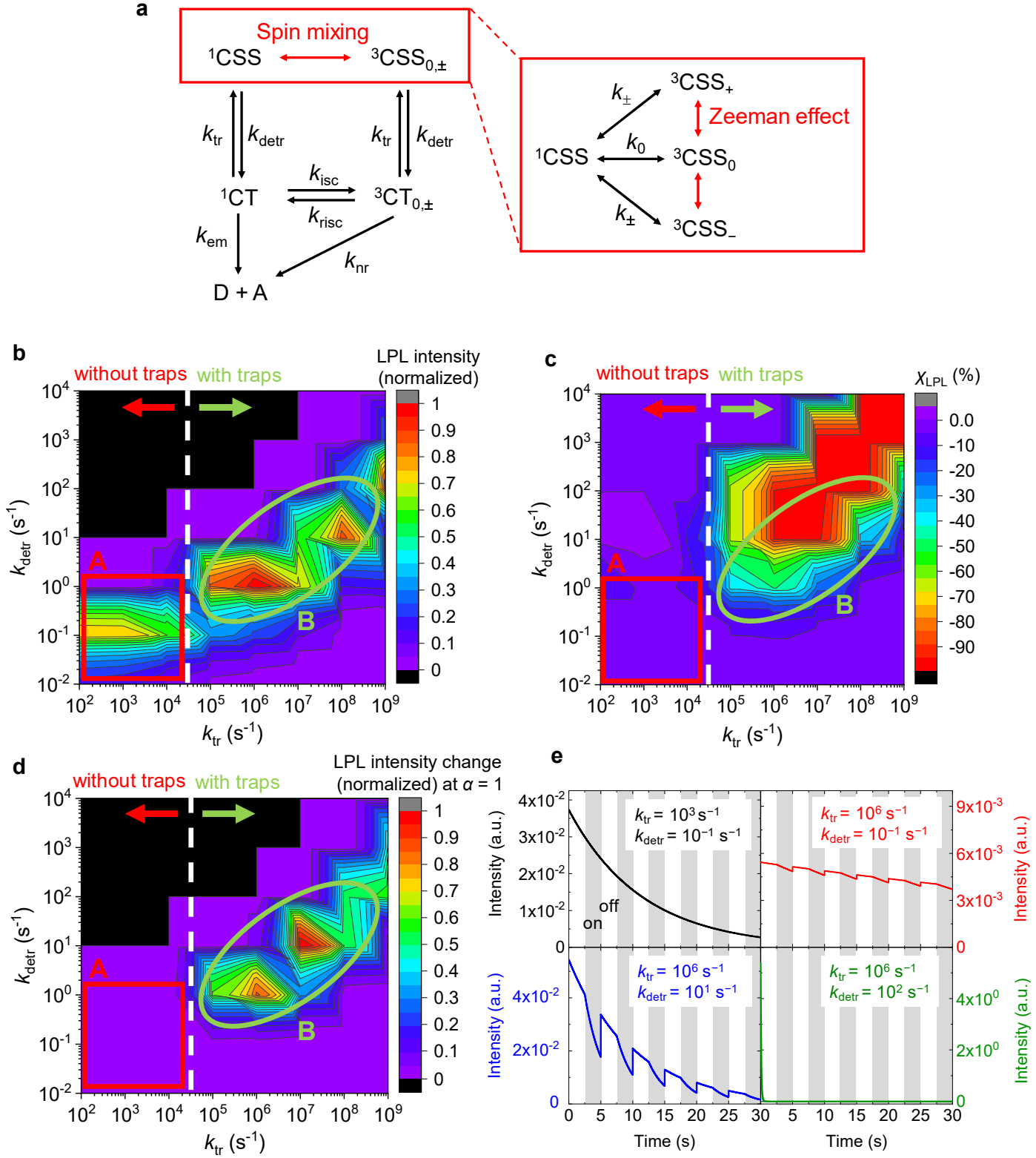

Extended Data Fig. 10. Simulations of MFEs on LPL. a, Reaction scheme. D is the donor, $\mathrm{A}$ is the acceptor, CT is the charge transfer, and CSS is the charge-separated state. $k_{\mathrm{em}}, k_{\text {isc }}, k_{\text {risc }}$, $k_{\mathrm{tr}}, k_{\mathrm{detr}} k_{\mathrm{nr}}, k_{0}$, and $k_{ \pm}$are the rates of emission, intersystem crossing, reverse intersystem crossing, trapping, detrapping, non-radiative transition, and spin-mixing between ${ }^{1} \mathrm{CSS}$ and ${ }^{3} \mathrm{CSS}_{0}$ or ${ }^{3} \mathrm{CSS}_{ \pm}$, respectively. b-d, Contour plots of (b) the LPL intensity, (c) $\chi_{\mathrm{LPL}}$, and (d) intensity change induced by the magnetic field after $10 \mathrm{~s}$ against $k_{\text {tr }}$ and $k_{\text {detr. }}$ e, Time traces of LPL intensity at $k_{ \pm}=0$ (gray regions) and $1.6 \times 10^{8}$ (white regions) $\mathrm{s}^{-1}$, which correspond with and without the magnetic field, respectively. 